The IJA is a peer-reviewed open-access, electronic journal, freely available without charge to users

Produced by the AquacultureHub non-profit Foundation Sale of IJA papers is strictly forbidden

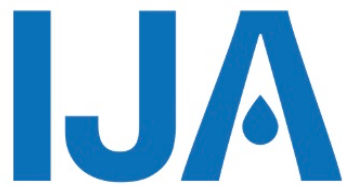

\title{
Difference analysis of gut microbiota of golden pompano Trachinotus ovatus (Linnaeus, 1758) with the unsynchronized growth by feeding fermented soybean meal
}

\author{
Bo Liu 1,2, Hua-Yang Guo 1,2, Bao-Suo Liu 1,2, Liang Guo 1,2, Nan \\ Zhang ${ }^{1,2}$, Ke-Cheng Zhu ${ }^{1,2}$, Qingquan Wang ${ }^{1}$, Dianchang Zhang \\ $1,2,3,4 *$
}

\begin{abstract}
${ }^{1}$ Key Laboratory of South China Sea Fishery Resources Exploitation and Utilization, Ministry of Agriculture and Rural Affairs; South China Sea Fisheries Research Institute, Chinese Academy of Fishery Sciences, 510300, Guangzhou, Guangdong Province, China

2 Tropical Aquaculture Research and Development Center, South China Sea Fisheries

Research Institute, Chinese Academy of Fishery Sciences, 572018, Sanya, Hainan Province, China

${ }^{3}$ Guangdong Provincial Engineer Technology Research Center of Marine Biological Seed Industry, Guangzhou, Guangdong Province, China

${ }^{4}$ Guangdong Provincial Key Laboratory of Fishery Ecology and Environment, Guangzhou, Guangdong Province, China

Keywords: Trachinotus ovatus, gut microbiota, biodiversity, different species
\end{abstract}

\begin{abstract}
Golden pompano (Trachinotus ovatus) is a commercially important marine fish and is widely cultured in the coastal area of South China. We explored the distribution difference of intestinal microflora with the same growth condition and the difference of gut microflora with different growth rates fed with fermented soybean meal (FSM) and fish meal (FM) primary protein source. After 8 weeks of the breeding experiment, select significant growth fish (Zcd, $156.04 \pm 1.04 \mathrm{~g})$ average growth fish $(Z \mathrm{c}, 120.01 \pm 1.05 \mathrm{~g})$ stagnant growth fish (ZcX, $98.03 \pm 1.08 \mathrm{~g})$ in FSM and select average growth fish (Dc, $158.03 \pm 1.02 \mathrm{~g}$ ) in FM. Sequencing results show that a total of 86303 sequences had been obtained in the intestine of Trachinotus ovatus. The number of OTUs that can be annotated to the database was 2315 (98.97\%). At the phylum level, Proteobacteria, Tenericutes, Spirochaetes and Firmicutes were the predominant phyla. Alpha diversity and beta diversity analyses show that gut microbes' richness and diversity in the DC group were the highest. The diversity of gut microbiota in the FSM: Zc > Zcd >Zcx. At the genus classification level, the differences between FM and FSM were mainly in Gueglia, Grimontia, Klebsiella, Alistipes, Staphylococcus, and Parasutterella $(p<0.05)$. Alistipes and Staphylococcus were the different genera between Dc and Zcd $(p<0.05)$. We found significant differences in the relative abundance of Ruegeria between the Zc and Zcd groups. Also, significant differences were found in the distribution of Brevinema, Ruegeria, Unidentified-Lachnospiraceae, Blautia, and Bacteroides between Zc and Zcx groups. The results showed that fermented soybean meal had an essential effect on gut microflora of $T$. ovatus with the unsynchronized growth.
\end{abstract}

* Corresponding author: Dr. Dian C. Zhang. 231 Xingang Road West, Haizhu District, Guangzhou City, Guangdong Province, 510300, PR China. E-mail address:

zhangdch@scsfri.ac.cn; Phone: +86 02089108316; fax: +86 02089022702 


\section{Introduction}

Golden pompano, Trachinotus ovatus, belongs to the family Carangidea (Liu et al., 2019), it is widely distributed in China's coastal provinces and the tropical and subtropical waters of southeast Asia and the Mediterranean (Guo et al., 2018). It has become an important fish in aquaculture in South China because of its advantages of wide temperature range, fast-growth speed, especially suitable for deep-sea anti-wind and wave cage culture (Xie et al., 2014). With the rapid development of aquaculture, fish meal resources are in short supply. As a supplement of fish meal (FM), industrial plant protein is widely used in some types of carnivorous fish feed (Ingerslev et al., 2014). Fermented soybean meal (FSM) is one of the sources of plant protein in aquatic feed. Many studies have confirmed that FSM can partially replace FM in Oncorhynchus mykiss (Barnes et al., 2012) and Acanthopagrus schlegelii (Zhou et al., 2011), and can obtain better growth performance and immune status.

Under normal circumstances, the gut microbiota of fish is in a dynamic balance state, and there is a competitive relationship between different flora in the intestinal tract. Meanwhile, the species and quantity of gut microbiota are also affected by the external environment (Gómez \& Balcázar, 2008). Daniel et al. (2014) found that gut microbiota was involved in the host's digestion and absorption of nutrients. Ingerslev et al. (2014b) found that fish gut microbiota's structural composition was highly correlated with food components. For example, Ingerslev et al. (2014a) showed that O. mykiss' intestine is colonized at an early state but is guided in new and different directions de-pendent on the diet type. Gut microbiota changed towards phylum Firmicutes dominance for plantbased fed fish and towards dominance of phylum Proteobacteria for the marine fed fish.

Physiology and disease were proposed based on the related changes in the composition and activity of the gut microbiota of fish, facilitating digestion and affecting nutrition, growth, reproduction, overall population dynamics, and host fish vulnerability diseases. Therefore, the microbial community is highly relevant to aquaculture practices (Ghanbari et al., 2015). For example, the number of harmful bacteria such as Aeromonas, Escherichia coli and Enterococcus in Ctenopharyngodon idellus intestines increased significantly, while the number of beneficial bacteria such as Lactobacillus and Bifidobacterium decreased, which will cause enteritis (Cerezuela et al., 2013).

In this study, 16S rDNA sequencing technology was used to sequence the gut microbiota of 4-golden pompano groups. By comparing the characteristics of gut microbiota in each group, the differences of gut microbiota in different protein sources on golden pompano were analyzed to explore whether beneficial bacteria can be added to the diet to maintain the relative balance of gut microbiota so as to improve the utilization rate of plant feed.

\section{Materials and Methods}

\section{Production of feed}

According to the formulated feed for gold pompano, the following two kinds of feed were prepared, a fish meal (FM) and a fermented soybean meal (FSM). The formula and nutrient composition are presented in Table $\mathbf{1}$. All the materials are purchased from Zhanjiang yinheng Biotechnology Co, Ltd. All materials are crushed and sieved for 60 mesh, and made with twin-screw granulator $(C D 4 \times 1 T S)$ and stored in a refrigerator at $20^{\circ} \mathrm{C}$ for standby

\section{Feeding management}

All golden pompano purchased from blue ocean aquaculture company, which were selected with the same body size and no damage on the body surface, 900-golden pompano (mean initial weight was $80.02 \pm 1.04 \mathrm{~g}$ ) were randomly allocated in 6 floating net cages. During the experiment, fish was fed to satiation at 9:00 am and 4:00 pm every day, lasted for 8 weeks. And the following water parameters were maintained: dissolved oxygen at $5.00 \mathrm{mg} / \mathrm{L}$, the temperature at $27.5-29.5^{\circ} \mathrm{C}$. 
Table 1 Feed formula and proximate analysis

\begin{tabular}{|c|c|c|}
\hline Composition & Fish meal & Fermented soybean meal \\
\hline Fish meal & 55 & 0 \\
\hline Fermented soybean & 0 & 57.5 \\
\hline Corn gluten & 0 & 25 \\
\hline Wheat bran & 32.5 & 0 \\
\hline Fish oil & 4 & 0 \\
\hline Soybean oil & 0 & 9 \\
\hline Alpha cellulose & 2 & 2 \\
\hline Soybean lecithin & 2 & 2 \\
\hline Dicalcium phosphate & 2 & 2 \\
\hline Choline chloride & 0.5 & 0.5 \\
\hline Vitamin premix & 1 & 1 \\
\hline Mineral premix & 1 & 1 \\
\hline Total & 100 & 100 \\
\hline Crude protein & 45.8 & 40.28 \\
\hline Crude lipid & 11 & 11 \\
\hline Water & 12.9 & 13.9 \\
\hline Ash & 12.3 & 5.7 \\
\hline
\end{tabular}

\section{Sample collection}

Before to sampling, golden pompano were stopped feeding for $24 \mathrm{~h}$, then the fish was sampled during anesthesia (MS-222). The fish meal group was marked as Dc, and the fermented soybean meal group was marked as Zc. Randomly select 9 fish from FM group $(158.03 \pm 1.02 \mathrm{~g})$, and quickly taken out their intestines and 3 fish mixed a sample stored at $-80^{\circ} \mathrm{C}$. Then according to the above steps, take samples of significant growth $(Z c, 156.04 \pm 1.04 \mathrm{~g})$, average growth (Zcd, $120.01 \pm 1.05 \mathrm{~g})$, stagnant growth (Zcx, $98.03 \pm 1.08 \mathrm{~g}$ ) in FSM group. All experiments in this study were approved by the Animal Care and Use Committee of South China Sea Fisheries Research Institute, Chinese Academy of Fishery Sciences (no. SCSFRI96-253), and performed according to the regulations and guidelines established by this committee.

\section{$16 S$ rDNA sequencing}

Total genome DNA from samples was extracted using CTAB/SDS method. DNA concentration and purity were monitored on $1 \%$ agarose gels. According to the concentration, DNA was diluted to $1 \mathrm{ng} / \mu \mathrm{L}$ using sterile water.16S rDNA genes of distinct regions (16S V3-V4) were amplified used a specific primer (16S V4: 515F-806R) with the barcode. All PCR reactions were carried out with $15 \mu \mathrm{L}$ of Phusion $\AA$ High-Fidelity PCR Master Mix (New England Biolabs); $0.2 \mu \mathrm{M}$ of forward and reverse primers, and about 10 ng template DNA. Thermal cycling consisted of initial denaturation at $98^{\circ} \mathrm{C}$ for $1 \mathrm{~min}$, followed by 30 cycles of denaturation at $98^{\circ} \mathrm{C}$ for $10 \mathrm{~s}$, annealing at $50^{\circ} \mathrm{C}$ for $30 \mathrm{~s}$, and elongation at $72^{\circ} \mathrm{C}$ for $30 \mathrm{~s}$. Finally, $72^{\circ} \mathrm{C}$ for $5 \mathrm{~min}$. Mix the same volume of $1 \mathrm{X}$ loading buffer (contained SYB green) with PCR products and operate electrophoresis on $2 \%$ agarose gel for detection. PCR products were mixed in equidensity ratios. Then, mixture of PCR products was purified with Qiagen Gel Extraction Kit (Qiagen, Germany). Sequencing libraries were generated usingTruSeq ${ }^{\circledR}$ DNA PCR-Free Sample Preparation Kit (Illumina, USA) following the manufacturer's recommendations and index codes were added. The library quality was assessed on the Qubit@ 2.0 Fluorometer (Thermo 
Scientific) and Agilent Bioanalyzer 2100 system. At last, the library was sequenced on an Illumina NovaSeq platform and 250 bp paired-end reads were generated.

\section{Data analysis}

Paired-end reads assembly and quality control. Paired-end reads were assigned to s amples based on their unique barcode and truncated by cutting off the barcode and prim er sequence. Paired-end reads were merged using FLASH (V1.2.7) (Magoc and Salzberg, 2011), a high-speed, and accurate analysis tool, which was designed to merge paired-en $\mathrm{d}$ reads when at least some of the reads overlap the read generated from the opposite en $\mathrm{d}$ of the same DNA fragment, and the splicing sequences were called raw tags. Quality filt ering on the raw tags was performed under specific filtering conditions to obtain the high -quality clean tags (Bokulich et al., 2013) according to the QIIME (V1.9.1) (Caporaso et a I., 2010) quality-controlled process. The tags were compared with the reference database (Silva database) using the UCHIME algorithm (UCHIME Algorithm) (Rognes et al., 2016) to detect chimera sequences, and then the chimera sequences were removed (Haas et al. , 2011). Then the Effective Tags were finally obtained.

OTU cluster and Species annotation. Sequence analyses were performed by Uparse software (Uparse v7.0.1001) (Edgar, 2013). Sequences with $\geq 97 \%$ similarity were assigned to the same OTUs (operational taxonomic units). Representative sequence for each OTU was screened for further annotation. For each representative sequence, the Silva Database (Wang et al., 2007) was used based on the Mothur algorithm to annotate taxonomic information. In order to study phylogenetic relationship of different OTUs, and the difference of the dominant species in different samples (groups), multiple sequence alignment was conducted using the MUSCLE software (Version 3.8.31) (Quast et al., 2012). OTUs abundance information was normalized using a standard of sequence number corresponding to the sample with the least sequences. Subsequent analyses of alpha diversity and beta diversity were all performed basing on this output normalized data.

Alpha diversity is applied in analyzing the complexity of species diversity for a sample through 6 indices, including Observed-species, Chao1, Shannon, Simpson, ACE, Good-coverage. All these indices in our samples were calculated with QIIME (Version 1.7.0) and displayed with $R$ software (Version 2.15.3). Principal component analysis (PCA) was applied to reduce the original variables' dimension using the FactoMineR package and ggplot2 package in $\mathrm{R}$ software (Version 2.15.3)

Statistical analyses. Data were reported as means \pm standard errors (SE) and oneway analysis of variance (ANOVA) followed by Tukey's multiple comparison tests was used to determine significant differences between treatments. All statistical analyses were performed using SPSS 22.0 (SPSS Inc., Chicago, USA). The significance level adopted was $95 \%(p<0.05)$.

\section{Results}

Sample sequencing results and diversity analysis

Based on the Illumina Nova sequencing platform, we sequenced 12-gut microbiota of golden pompano. The average of each sample was 86303 sequences. The compelling data of quality control was 61759 , and the effective rate of quality control was $72.36 \%$. The sequencing information is presented in Table 2.

The OTUs sequence was clustered into OTUs with $97 \%$ identity, and species annotation was carried out between OTUs sequence and silva132 database, and statistics of different classification levels were made. In this study, 2339 OTUs were obtained, among which $2315(98.97 \%)$ could be annotated to the database, $81.57 \%$ could be annotated to the Phylum level, $78.50 \%$ could be annotated to the class level, $72.21 \%$ could be annotated to the Order level, $65.07 \%$ could be annotated to the family level, $44.21 \%$ could be annotated to the genus level and $14.24 \%$ could be annotated to the species. There were 971 OTUs be annotated in the Dc group, 906 OTUs in the Zc group, 869 OTUs in the Zcd group and 621 OTUs in the Zcx group. The OTUs of each sample 
were statistically analyzed, and a Venn diagram was constructed (Figure 1). It can be seen that there were 663 identical OTUs in 4 groups $(34.67 \%$ of the total OTUs), Dc group had the greatest number of unique OTUs (195).

Table 2 Sequencing information

\begin{tabular}{llllll}
\hline Name & Raw PE & Effective Tags & Base (nt) & AvgLen (nt) & Effective (\%) \\
\hline Dc1 & 89716 & 62406 & 26406609 & 423 & 69.56 \\
Dc2 & 84505 & 61607 & 26122743 & 424 & 72.9 \\
Dc3 & 95984 & 61264 & 26042757 & 425 & 63.83 \\
Zc1 & 81603 & 63475 & 26768656 & 422 & 77.79 \\
Zc2 & 96174 & 61857 & 26307924 & 425 & 64.32 \\
Zc3 & 70208 & 54468 & 22800808 & 419 & 77.58 \\
Zcd1 & 81884 & 65688 & 27719950 & 422 & 80.22 \\
Zcd2 & 97268 & 62691 & 26591904 & 424 & 64.45 \\
Zcd3 & 98406 & 63638 & 26997407 & 424 & 64.67 \\
Zcx1 & 92432 & 61719 & 26291628 & 426 & 66.77 \\
Zcx2 & 69421 & 60054 & 25351194 & 422 & 86.51 \\
Zcx3 & 78032 & 62244 & 26427174 & 425 & 79.77 \\
\hline
\end{tabular}

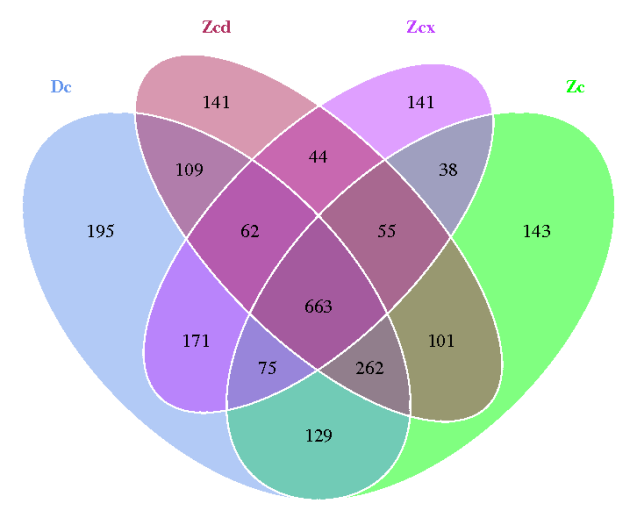

Figure 1 Venn diagram analysis of intestine microbial with OTUs of $T$. ovatus

The alpha diversity analysis indexes are presented in Table 3, different samples under the $97 \%$ consistency threshold were statistically analyzed. ACE, Chao1, Shannon and Simpson diversity indices were no significant difference in each group.

The Rarefaction Curve drawn by OTUs indicates that the sequencing depth of the bacterial community structure has become saturated, and the experimental samples are sufficient to reflect the microbial diversity contained in the community samples (Figure 2). The Rank Abundance can intuitively see the species richness and species distribution uniformity of each group (Figure 3). The intestinal bacteria richness was highest in the 
Dc group, and the Zc group has the most distribution of gut microbiota. The Zcd group's gut microbiota's abundance and uniformity were significantly lower than those of the Dc and Zc group but better than the Zcx group.

Table 3 Alpha diversity analysis indexes

\begin{tabular}{llllll}
\hline Sample & Observed species & Shannon & Simpson & Chao1 & ACE \\
\hline Dc & $970.67 \pm 292$ & $4.343 \pm 0.5$ & $0.78 \pm 0.08$ & $1100 \pm 317$ & $1105.41 \pm 318$ \\
Zc & $906 \pm 198$ & $4.443 \pm 0.9$ & $0.78 \pm 0.07$ & $1255 \pm 407$ & $1153.54 \pm 370$ \\
Zcd & $869.33 \pm 130$ & $3.4 \pm 0.4$ & $0.78 \pm 0.07$ & $1005 \pm 190$ & $1041.45 \pm 83$ \\
Zcx & $621.33 \pm 152$ & $2.95 \pm 0.3$ & $0.71 \pm 0.04$ & $710 \pm 194$ & $719.87 \pm 198$ \\
\hline
\end{tabular}

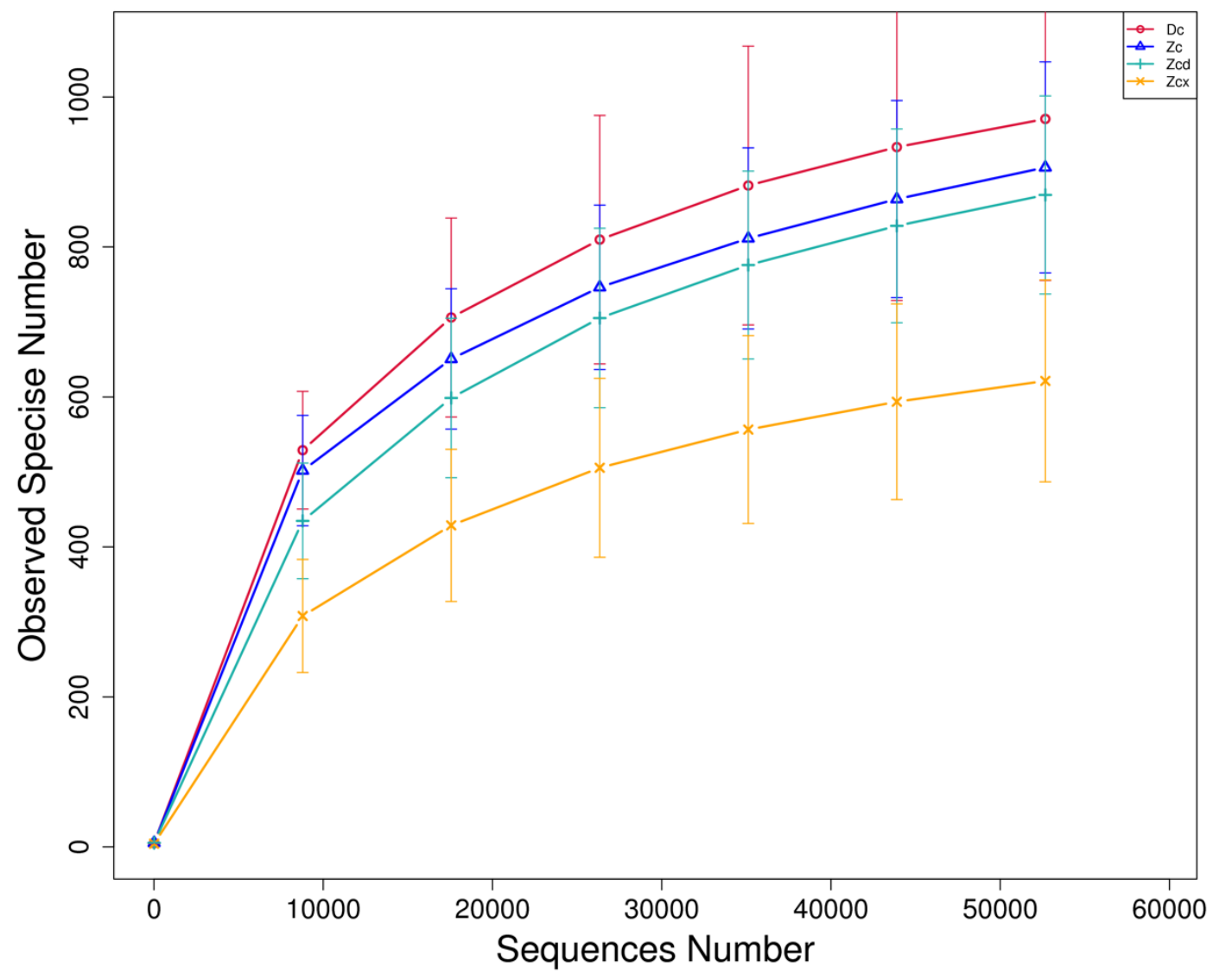

Figure 2 Rarefaction curve of intestinal microbial diversity of $T$. ovatus 


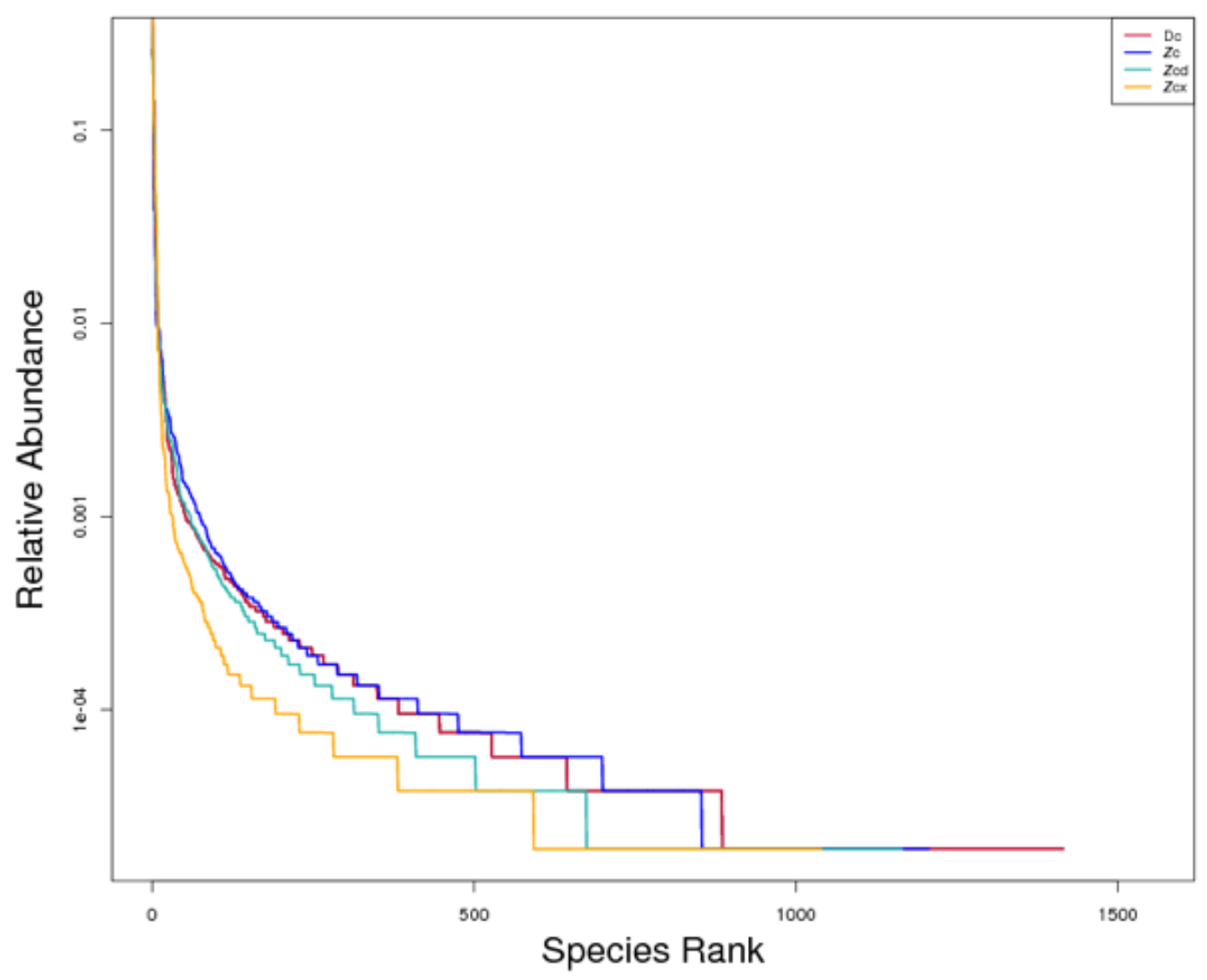

Figure 3 Rank Abundance curve of intestinal microbial diversity of T. ovatus

\section{Species distribution}

Distribution difference of gut microbiota at phylum level. By comparing the relative a bundance

of gut microbiota (the top 10 species with the most significant relative abundance) at the phylum level of each group of samples (Figure 4), we found that the dominant species were Proteobacteria, Tenerictes, Spirochaetes, Firmicutes, and Bacteroidetes. The results showed that the species distribution of intestinal flora at the phylum level were not different in each group, but there were significant differences in the number distribution. The results showed that there were significant differences in Actinobacteria and Gemmatimondetes between the Dc and Zcd. There were significant differences in Spirochaetes between the Zcd and Zcx. There were significant differences in Spirochaetes and Firmicutes between the Zc and Zcx $(p<0.05)$. According to Figure 5, it can be seen that Proteobacteria is widely distributed in FSM, but more distributions in the Zcd and Zcx group, while Tenericutes and Firmicutes are less distributed in the Zcx group and have obvious differences in dominance in each group. 


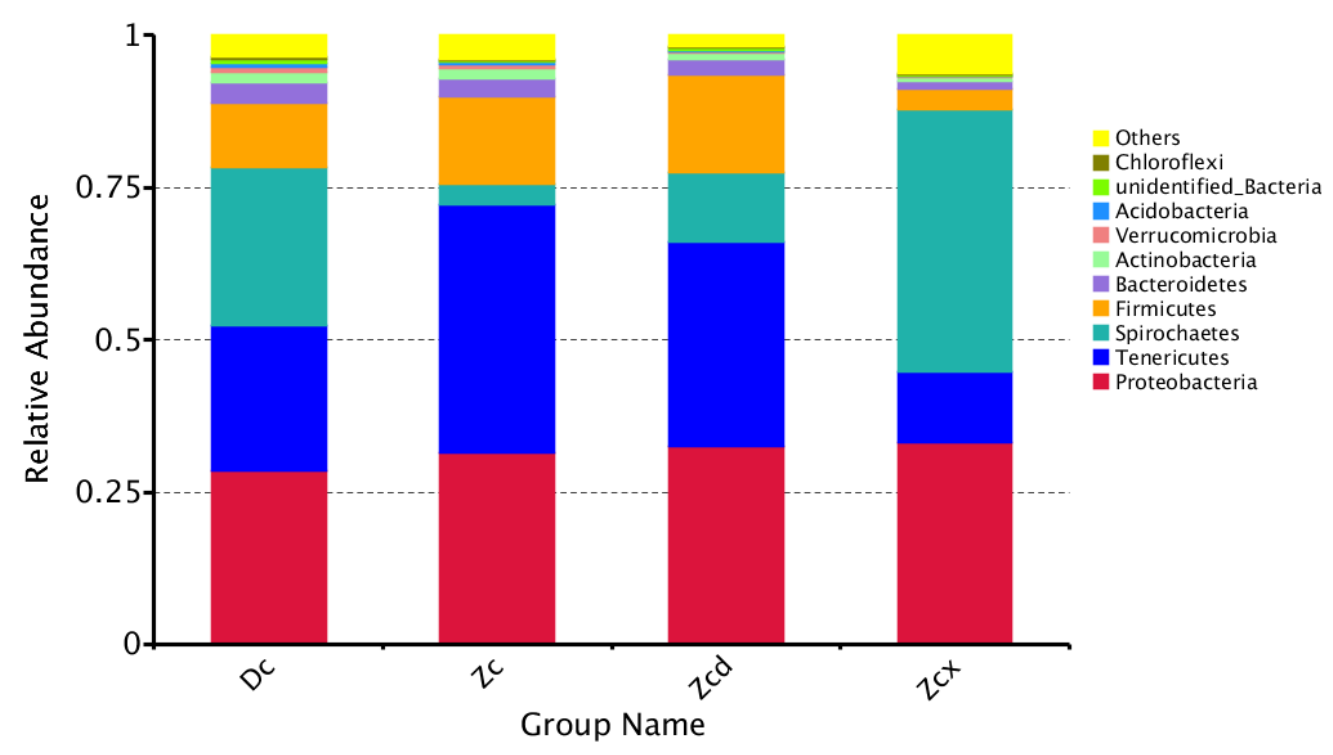

Figure 4 Bacterial relative abundance in intestine of $T$. ovatus at phylum level

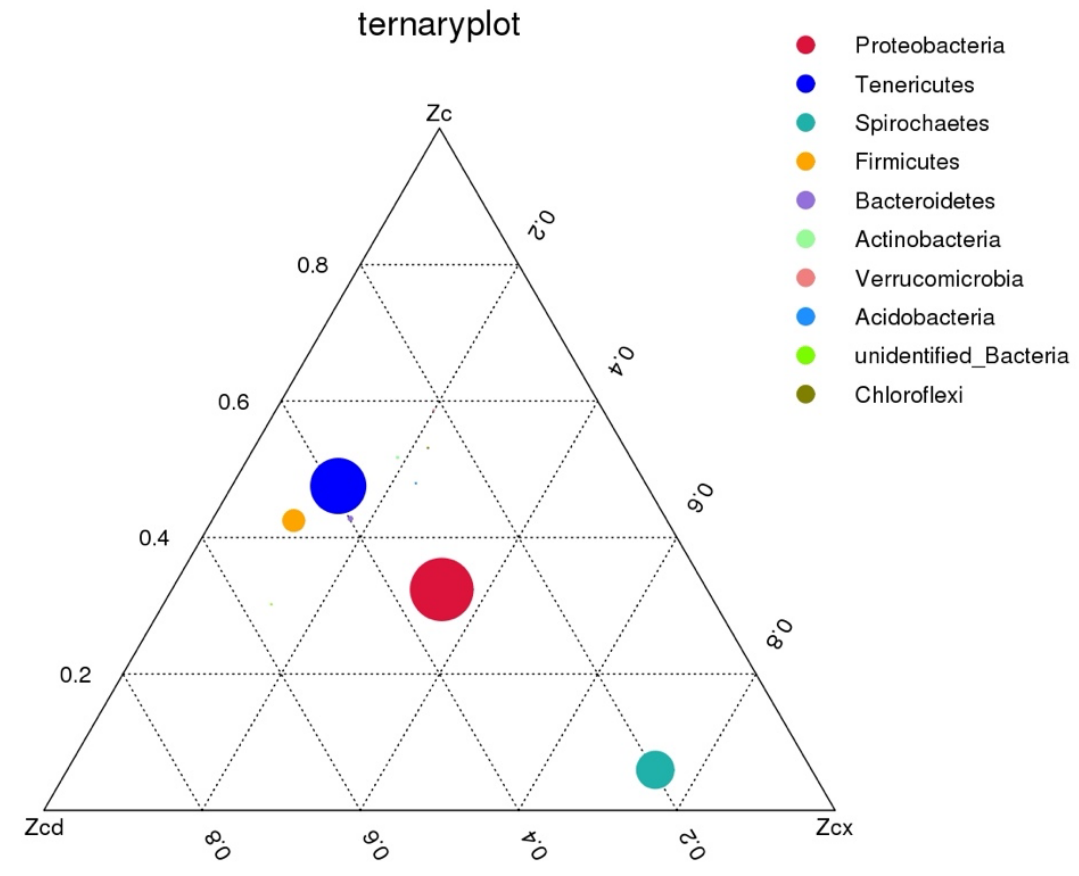

Figure 5 Ternaryplot in intestine of $T$. ovatus at phylum level

Distribution difference of gut microbiota at genus level. By looking for the relative abundance of gut microbiota at the genus level of each group of samples (the top 35 species with the most significant relative abundance) (Figure 6), the dominant species at the genus level were Mycoplasma, Brevinema, and Photobacterium. 


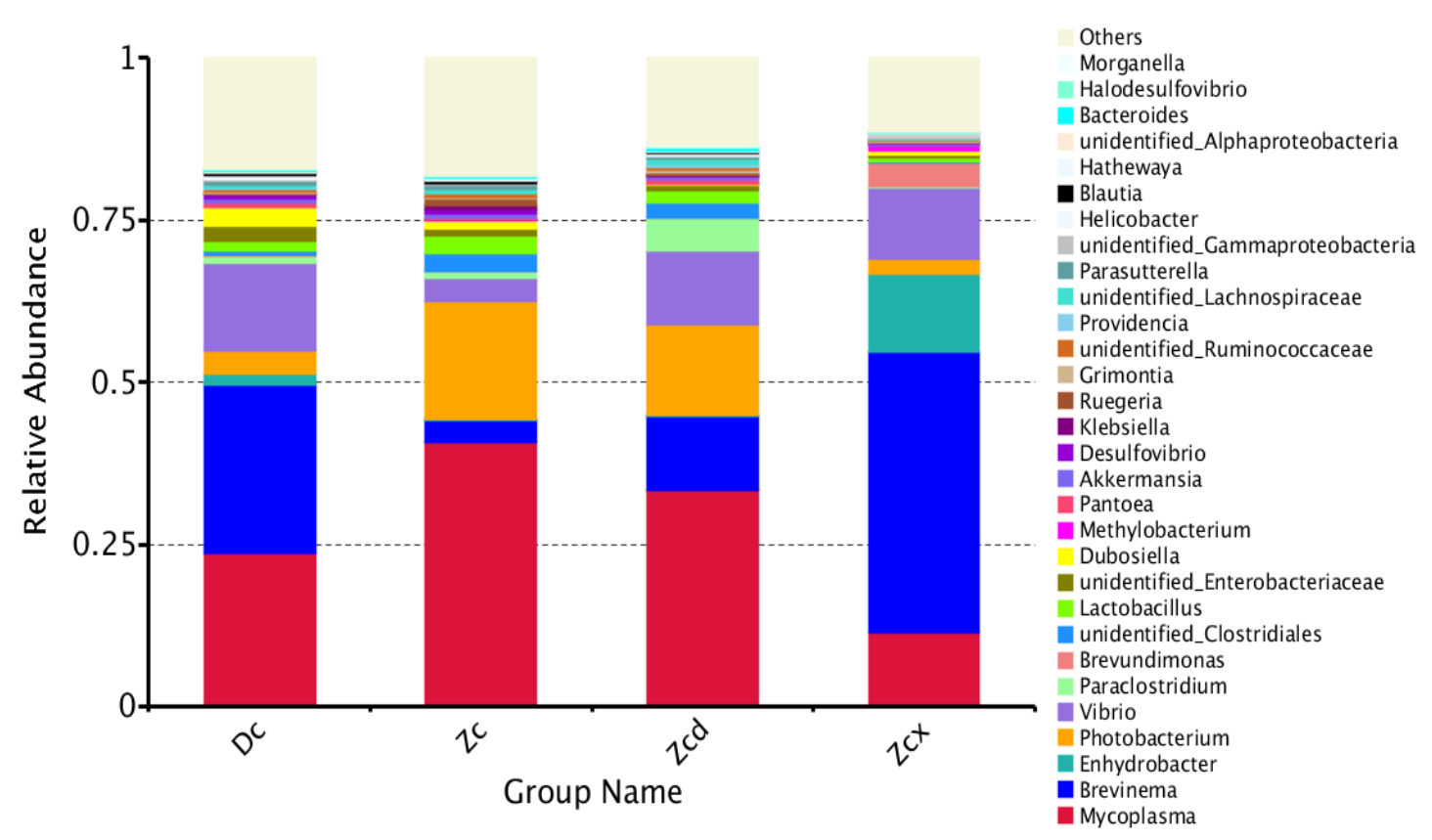

Figure 6 Bacterial relative abundance in intestine of $T$. ovatus at genus level

The results showed: there were significant differences in Ruegiria, Grimontia, Alistipes between the Dc and the Zcd at the level of genera; There were significant differences in Alistipes, Staphylococcus between the Dc and the Zcd; there were significant differences in Klebsiella, Parasutterella, Alistipes between the Dc and the Zcx $(p<0.05)$; Brevinema and Bacteroides were significantly different in the Zcd and the Zcx $(p<0.05)$. There were significant differences in Ruegiria between the Zc and the Zcd $(p<0.05)$; Brevinema, Ruegiria, Blautia, Bacteroides and Unidentified-Lachnospiraceae were significant differences between the Zc and the Zcx $(p<0.05)$. It could be seen that the dominance distribution of Mycoplasma, Photobacterium, Unidentified-Clostridiales and Vibrio was Zc $>$ Zcd $>$ Zcx, while the distribution of Brevinema and Vibrio was Zc $<$ Zcd $<$ Zcx (Figure 7). 


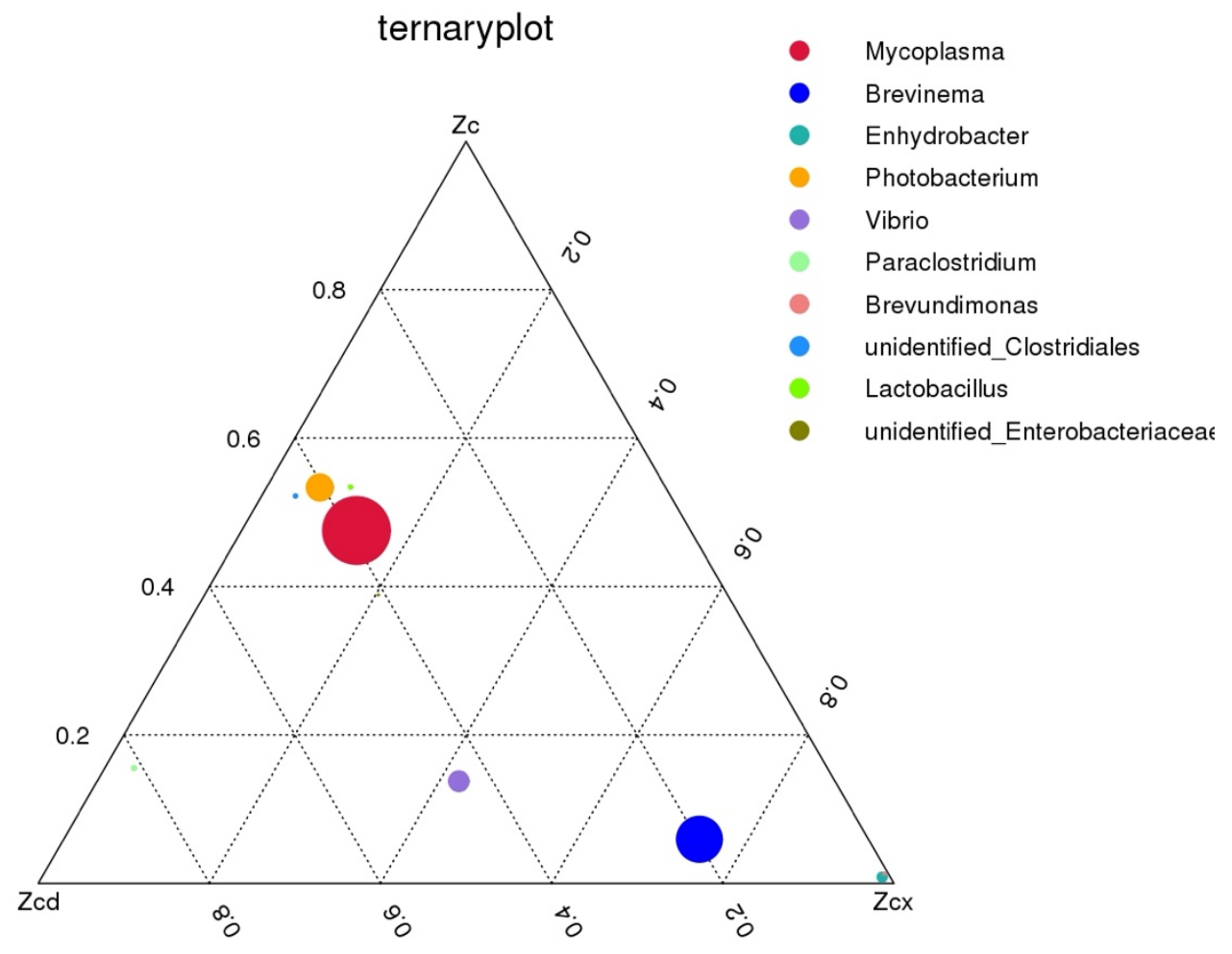

Figure 7 Ternaryplot in intestine of $T$. ovatus at genus level

PCA analysis. Based on the best classification level, the principal component analysis of each sample's microbial community structure was carried out to observe the relationsh ip between each sample (Figure 8). It can be seen that the intestinal microbiota of FSM were different, and the structure of the intestinal microbiota of the Zc and the Zcd groups have higher similarity. However, the intestinal microbiota distribution in the Zcx group w as irregular, indicating that the microbiota was quite different. 


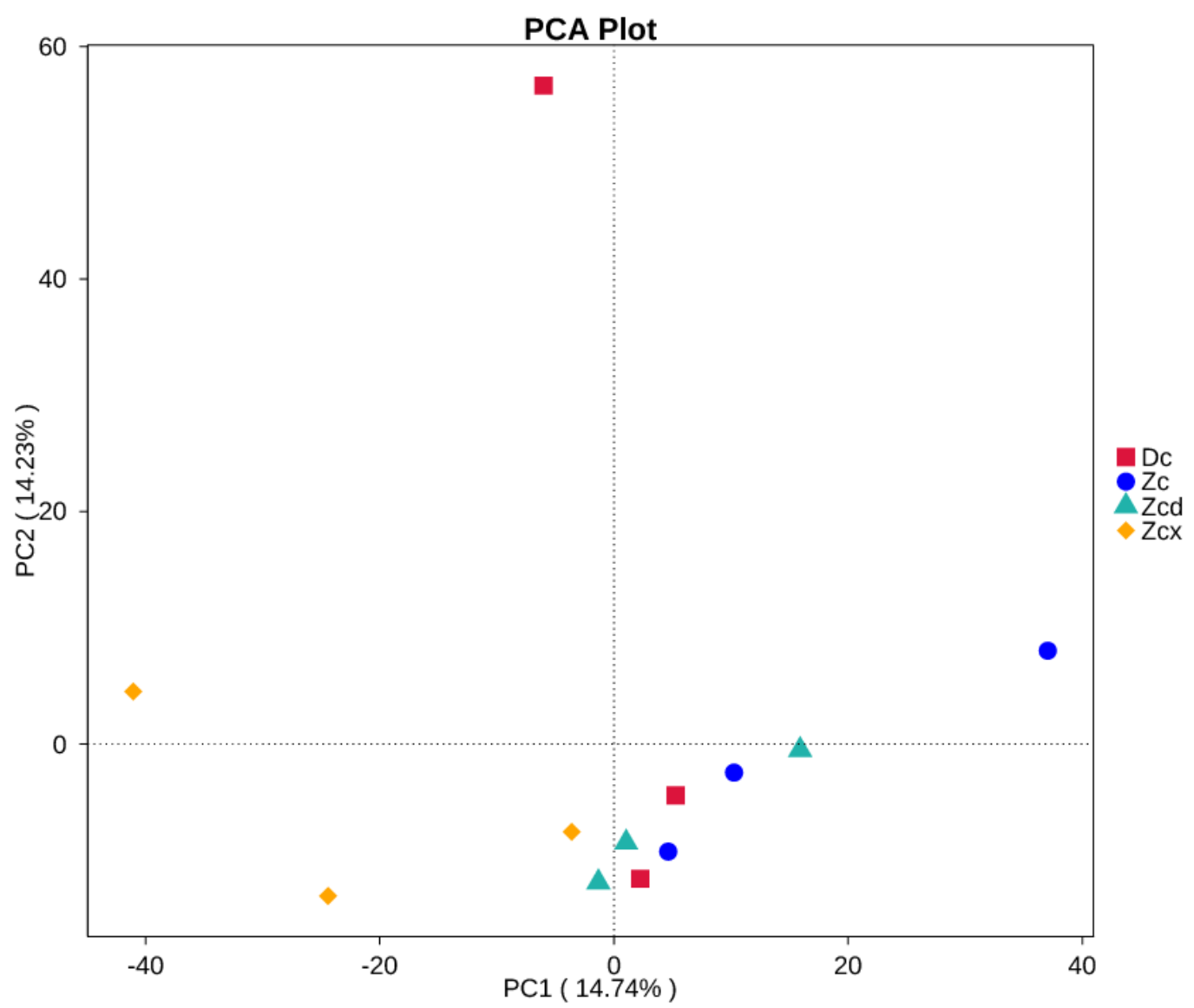

Figure 8 Principal co-ordinates analysis of intestinal microbiota in T. ovatus

\section{Discussion}

In this study, we compared the effects of FSM on the gut microbiota of individuals with unsynchronized growth of golden pompano. The results showed that the average OTUs number of FM was more significant than that of FSM. We speculate that golden pompano needs more intestinal microbiota to digest omnivorous feed than vegetarian feed. Larsen et al. (2014) have been studied intestinal microbiota in fish of different feeding habits and showed that the number of gut microbiota of omnivorous fish was significantly greater than that of carnivorous fish. Our study showed that the Shannon, Chao1, and ACE indexes in the Dc group were smaller than those of the Zc group, indicating that the diversity of gut microbiota in the golden pompano feed on a vegetarian diet was more significant than that on an omnivorous diet, which was similar to the results of Scophthalmus maximus (Givens et al., 2015). The number of Shannon, Chao1, and ACE indexes of the individuals with unsynchronized growth in FSM feed were all following the rule of Zc > Zcd > Zcx, which indicated that the diversity of gut microbiota was related to the growth of golden pompano.

According to species annotation results, the dominant bacteria in each group at the phylum level were Proteobacteria, Firmicutes, Bacteroidetes and Spirochaetes, which were consistent with the results of other fish (Wei et al., 2017; Irianto \& Austin, 2002). We found that the stable distribution of Proteobacteria in each group was $27 \%$, indicating that the dominant intestinal flora had colonization stability (Yu et al., 2015). While other phyla were significantly different in the experimental group, Spirochaetes and Tenericutes are basically the same in the Dc group, but Tenericutes content is significantly higher than Spirochaetes in the Zcd group and has a tendency to shift. There may be a complex ecological relationship between these two bacterial communities that inhibits each other (Lagier et al., 2004). In a comparison of Firmicutes content, Zcx 
group significantly reduced of Zc and Zcd group. Bairagi et al. (2015) showed that the increase of Firmicutes was conducive to the acquiring nutrients and weight gain of the host. Studies have shown that Firmicutes play an essential role in stimulating host metabolism and increasing the bio-availability of fatty acids by modifying bile salts (Semova et al., 2012). Chapagain et al. (2019) showed that some bacterial taxa with functional implications indicate of fish growth rate. Therefore, the stagnant growth of the Zcx group may be related to Firmicutes.

On genus level, Rueglia, Grimontia, Klebsiella, Alistipes, Staphylococcus, and Parasutterella have significant differences in FM and FSM group. Staphylococcus, and Parasutterella are kind of bacteria with digestive function, has not been reported about gut microbiota of fish, and further studies are needed. Alistipes is the only different genus between all groups. Therefore, it is deduced that Alistipes play an vital role in the digestion of FSM. Zcx group has significant differences in Brevinema, Ruegiria, Unidentified-Lachnospiraceae and Blautia with Zcd and Zc, but Brevinema belongs to pathogenic bacteria (Defosse et al., 1995). Studies have shown that Salmo salar enteritis may be related to a plant-based diet (Marjara et al., 2012). Therefore, enteropathy may be one of the reasons for the instability of gut microbiota in Zcx. Ruegiria is only in ecological function and water distribution characteristics, but research on gut microbiota has not been reported. Unidentified-Lachnospiraceae and Blautia belong to Firmicutes phylum, the content of which has certain influence on the digestion of host nutrients.

\section{Conclusion}

Fish meal and fermented soybean meal did not affect the diversity of intestinal bacteria in golden pompano, but the dominant species were different. The fermented soybean meal influences the gut microflora of golden pompano, which has unsynchronized growth. Alistipes in Bacteroidia and unidentified-Lachnospiraceae, Blautia in Firmicutes positively affect the fermented soybean meal's digestion on golden pompano. They play a vital role in improving the utilization rate of plant protein.

\section{Acknowledgments}

This work was supported by the Central Public-interest Scientific Institution Basal Research Fund CAFS (NO.2020TD29), China Agriculture Research System (CARS-47), Financial Fund of Ministry of Agriculture and Rural Affairs of China (NHYYSWZZZYKZX2020), Key Special Project for Introduced Talents Team of Southern Marine Science and Engineering Guangdong Laboratory (Guangzhou) (GML2019ZD0605), Hainan Provincial National Science Foundation of China (320QN363).

\section{References}

Bairagi A., Sarkar G.K., Sen S.K., Ray A.K., 2015. Evaluation of the nutritive value of leucaena leucocephala leaf meal, inoculated with fish intestinal bacteria bacillus subtilis and bacillus circulans in formulated diets for rohu, Labeo rohita (Hamilton) fingerlings. Aquac Res, 35, 436-446. https://doi.org/10.1111/j.13652109.2004.01028.x

Barnes M.E., Brown M.L., Rosentrater K.A., Sewell J.R., 2012. An initial investigation replacing fish meal with a commercial fermented soybean meal product in the diets of juvenile rainbow trout. Open Journal of Animal Sciences, 2(4), 234-243. https://doi.org/10.4236/ojas.2012.24033

Bokulich N.A., Subramanian S., Faith J.J., Gevers D., Gordon J.I., Knight R., Mills D.A., Caporaso J.G. 2013 Quality-filtering vastly improves diversity estimates from illumina amplicon sequencing. Nat Methods, 10, 57-59. https://doi.org/10.1038/nmeth.2276

Bulgarelli D., Garrido-Oter R., Münch P.C., Weiman A., Dröge J., Pan, Y., 2015. Structure and function of the bacterial root microbiota in wild and domesticated

barley. Cell Host
https://doi.org/10.1016/j.chom.2015.01.011 
Caporaso J.G., Kuczynski J., Stombaugh J., Bittinger K., Bushman F.D., Costello E.K., 2010. Qiime allows analysis of high-throughput community sequencing data. Nat Methods, 7, 335-336. https://doi.org/10.1038/nmeth.f.303

Cerezuela R., Fumanal M., Tapia-Paniagua S.T., Meseguer J., Moriñigob M.A., Estebana M., 2013. Changes in intestinal morphology and microbiota caused by dietary administration of inulin and bacillus subtilis in gilthead sea bream (Sparus aurata L.) specimens. Fish Shellfish Immunol, 34, 1063-1070. https://doi.org/10.1016/j.fsi.2013.01.015

Chapagain P., Arivett B., Cleveland B.M., Walker D.M., Salem M., 2019. Analysis of the fecal microbiota of fast- and slow-growing rainbow trout (Oncorhynchus mykiss). BMC Genomics, 20, 788. https://doi.org/10.1186/s12864-019-6175-2

Daniel H., Desmarchelier, C., Gholami A.M., Hahne H., Berry D., Böhm C., 2014. High-fat diet alters gut microbiota physiology in mice. ISME J, 8, 295-308. https://doi.org/10.1038/ismej.2013.155

Defosse D.L., Johnson R.C., Paster B.J., Dewhirst F.E., Fraser G.J., 1995. Brevinema andersonii gen. nov., sp. nov., an infectious spirochete isolated from the short-tailed shrew (Blarina brevicauda) and the white-footed mouse (Peromyscus leucopus). Int J Syst Bacteriol, 45, 78-84. https://doi.org/10.1099/00207713-45-1-78

Edgar R.C., 2013. Uparse: highly accurate otu sequences from microbial amplicon reads. Nat Methods, 10, 996-998. https://doi.org/10.1038/nmeth.2604

Ghanbari M., Kneifel W., Domig K.J., 2015. A new view of the fish gut microbiome: advances from next-generation sequencing. Aquaculture, 448, 464-475. https://doi.org/10.1016/j.aquaculture.2015.06.033

Givens C.E., Ransom B., Bano N., Hollibaugh J.T., 2015. Comparison of the gut microbiomes of 12 bony fish and 3 shark species. Marine Ecology Progress, 518, 209-223. https://doi.org/10.3354/meps11034.

Gómez G.D., Balcázar J.L., 2008. A review on the interactions between gut microbiota and innate immunity of fish. FEMS Immunol Med Mic, 52, 145-154. https://doi.org/10.1111/j.1574-695X.2007.00343.X

Guo L., Zhang N., Yang J.W., Guo H.Y., Zhu K.C., Liu B.S., Liu T.T., Zhang D.C., 2018. Comprehensive assessment of the genetic diversity and population structure of cultured populations of golden pompano, Trachinotus ovatus (linnaeus, 1758) by microsatellites. Aquacult Int, 26, 1445-1457. https://doi.org/10.1007/s10499-0180289-4

Haas B.J., Gevers D., Earl A.M., Feldgarden M., Ward D.V., Giannoukos G., 2011. Chimeric 16s rRNA sequence formation and detection in sanger and 454pyrosequenced pcr amplicons. Genome Res, 21, 494-504. https://doi.org/10.1101/gr.112730.110.

Ingerslev H.C., Jørgensen L., Strube M.L., Larsenc N., Dalsgaarda I., Boyea M., Madsena L., 2014a. The development of the gut microbiota in rainbow trout (Oncorhynchus mykiss) is affected by first feeding and diet type. Aquaculture, 424425, 24-34. https://doi.org/10.1016/j.aquaculture.2013.12.032.

Ingerslev H.C., Strube M.L., Jørgensen L., Dalsgaard I., Boye M., Madsen L., 2014b. Diet type dictates the gut microbiota and the immune response against Yersinia ruckeri in rainbow trout (Oncorhynchus mykiss). Fish Shellfish Immunol, 40, 624-633. https://doi.org/10.1016/j.fsi.2014.08.021

Irianto A., and Austin B., 2002. Probiotics in aquaculture. Journal of Fish Diseases, 25, 633-642. https://doi.org/10.1016/j.dit.2013.03.003.

Lagier M.J., Joseph L.A., Passaretti T.V., Musser K.A., Cirino N.M., 2004. A realtime multiplexed pcr assay for rapid detection and differentiation of Campylobacter jejuni and Campylobacter coli. Mol Cell Probes, 18, 275-282. https://doi.org/10.1016/j.mcp.2004.04.002

Larsen A.M., Mohammed H.H., Arias C.R., 2014. Characterization of the gut microbiota of three commercially valuable warmwater fish species. $J$ Appl Microbiol, 116, 1396-1404. https://doi.org/10.1111/jam.12475. 
Liu B., Guo H.Y., Zhu K.C., Guo L., Liu B.S., Zhang N., Jiang S.G., Zhang D.C., 201 9. Salinity Effect on Intestinal Microbiota in Golden Pompano Trachinotus Ovatus (L innaeus, 1758). ISR J AQUACULT-BAMID, 71, 1538. https://doi.org/10.46989/001c 20995

Magoc T., Salzberg S.L., 2011. Flash: fast length adjustment of short reads to improve genome assemblies. Bioinformatics, 27,

2957-2963. https://doi.org/10.1093/bioinformatics/btr507.

Marjara I.S., Chikwati E.M., Valen E.C., Krogdahl A., Bakke A.M., 2012. Transcriptional regulation of il-17a and other inflammatory markers during the development of soybean meal-induced enteropathy in the distal intestine of atlantic salmon (Salmo salar L.). Cytokine, 60, 186-196. https://doi.org/10.1016/j.cyto.2012.05.027

Quast C., Pruesse E., Yilmaz P., Gerken J., Schweer T., Yarza P., Peplies J., Glöckner F.0., 2013. The SILVA ribosomal RNA gene database project: improved data processing and web-based tools. Nucleic Acids Res, 41, 590-596. https://doi.org/10.1093/nar/gks1219

Rognes T., Flouri T., Nichols B., Quince C., Mahé F., 2016. Vsearch: a versatile open-source tool for metagenomics. Peer J, 4, e2584. https://doi.org/10.7717/peerj.258

Semova I., Carten J.D., Stombaugh J., Mackey L.C., Knight R., Farber S.A., Rawls J.F., 2012. Microbiota regulate intestinal absorption and metabolism of fatty acids in the zebrafish. Cell Host Microbe, 12, 277-288. https://doi.org/10.1016/j.chom.2012.08.003

Wang Q., Garrity G. M., Tiedje J.M., Cole J.R., 2007. Naive bayesian classifier for rapid assignment of rRNA sequences into the new bacterial taxonomy. Appl Environ Microb, 73, 5261-5267. https://doi.org/10.1128/AEM.00062-07

Wei N., Wang C., Xiao S., Huang W., Ma Y., 2017. Intestinal microbiota in large yellow croaker, Larimichthys crocea, at different ages: intestinal microbiota of large yellow croaker. J World Aquacult Soc, 10, 1111. https://doi:10.1111/jwas.12463

Xie Z.Z., Ling X., Wang D.D., Fang C., Liu Q.Y., Li Z.H., Liu X.C., Zhang Y., Li S.S., Lin H.R., 2014. Transcriptome Analysis of the Trachinotus ovatus: Identification of Reproduction, Growth and Immune-Related Genes and Microsatellite Markers. PloS one, 9. https://doi.org/10.1371/journal.pone.0109419.t001

Zhou F., Song W., Shao Q., Peng X., Xiao J.X., Hua Y., Owari B.N., Zhang T.Z., Ng W.K., 2011. Partial Replacement of Fish Meal by Fermented Soybean Meal in Diets for Black Sea Bream, Acanthopagrus schlegelii, Juveniles. J World Aquacult Soc, 42(2), 184-197. https://doi.org/10.1111/j.1749-7345.2011.00455.x 